\title{
A Method Based on Numerical Wind Field and Extreme Learning Machine for Typhoon Wind Speed Prediction of Wind Farm
}

\author{
Hong $X u^{1}$ and Wan-Yu Wang $\mathbb{D}^{2}$ \\ ${ }^{1}$ State Energy Jilin Jiangnan Thermal Power Co., Ltd, Jilin 132000, China \\ ${ }^{2}$ School of Economy and Management, North China Electric Power University, Beijing 102206, China \\ Correspondence should be addressed to Wan-Yu Wang; wwy20142021@163.com
}

Received 25 June 2021; Revised 20 October 2021; Accepted 26 October 2021; Published 20 November 2021

Academic Editor: Hongguang Pan

Copyright (c) 2021 Hong Xu and Wan-Yu Wang. This is an open access article distributed under the Creative Commons Attribution License, which permits unrestricted use, distribution, and reproduction in any medium, provided the original work is properly cited.

\begin{abstract}
Typhoon wind speed prediction is of great significance for it can help prevent wind farms from damages caused by frequent typhoon disasters in coastal areas. However, most researches on wind forecast are either for meteorological application or for normal weather. Therefore, this paper proposes a systematic method based on numerical wind field and extreme learning machine for typhoon wind speed prediction of wind farms. The proposed method mainly consists of three parts, IGA-YanMeng typhoon numerical simulation model, typhoon status prediction model, and wind speed simulation model based on an extreme learning machine. The IGA-YanMeng typhoon numerical simulation model can greatly enrich typhoon wind speed data according to historical typhoon parameters. The typhoon status prediction model can predict the status of typhoons studied in the next few hours. And wind speed simulation model simulates the average wind speed magnitude/direction at $10 \mathrm{~m}$ height of each turbine in the farm according to the predicted status. The end of this paper presents a case study on a wind farm located in Guangdong province that suffered from the super typhoon Mangkhut landed in 2018. The results verified the feasibility and effectiveness of the proposed method.
\end{abstract}

\section{Introduction}

Wind power has become a hot spot because it is friendly to the environment and it can supply a huge amount of energy for human society. More and more countries pay attention to wind power and build wind farms in the coastal area. However, frequent typhoon disasters have always been a huge threat to the normal operation of wind turbines. As a result, typhoon wind speed prediction deserves more concern.

At present, researches on wind forecast for wind farms in recent years can mainly be divided into two categories. One is a meteorological forecast of typhoon weather, which focuses on forecasting the meteorological data of typhoon. Yuan et al. [1] built up a model based on long short-time memory to forecast the typhoon intensity. Some studies tried to find out factors that influence the typhoon intensity forecast, such as the uncertainty of boundary layer [2] and lightning data [3]. Forecasting rainfall, flood, and storm surge ensemble along typhoon is also concerned via approaches such as GPU [4], radar [5, 6], and artificial neural network $[7,8]$. What's worth noting is that these types of meteorological models require a huge amount of calculation, which makes it difficult to be used for engineering application.

The other category is wind speed forecast under ordinary weather. Great progress has been achieved in this field with many mature technologies utilized such as artificial neural network [9, 10], ARIMA [11], support vector machine [12], and sample clustering [13]. And some researches are combined with meteorological technology such as numerical weather prediction $[14,15]$. However, few studies applied for engineering emphasize wind speed forecast under typhoon weather. One of the big problems with this issue is the lack of measured typhoon data. For wind speed under ordinary weather, massive measured data can be recorded every day. 
But it is different when it comes to typhoon data. For a certain local area, only a small number of typhoons occur and will be recorded.

Therefore, this paper proposes a systematic method based on numerical wind field and extreme learning machine for typhoon wind speed prediction suitable for the engineering field, alleviating the problem of huge calculation and the lack of measured typhoon data. Section 2 introduces the framework of the method proposed as a whole. It mainly consists of three parts: IGA-YanMeng typhoon numerical simulation model, typhoon status prediction model, and wind speed simulation based on extreme learning machine. Section 3 introduces how the IGA-YanMeng typhoon numerical simulation model simulates the wind speed (including magnitude and direction) of each wind turbine under historical typhoons selected. And Section 4 introduces the typhoon status prediction model, whose purpose is to predict the status of typhoon studied in the next 1 hour to 6 hours. Then Section 5 introduces the wind speed simulation model based on ELM and how it simulates the average wind speed magnitude/direction at $10 \mathrm{~m}$ height of each turbine in the farm according to the predicted status. In Section 6, a case study on a wind farm suffering from the super typhoon Mangkhut in 2018 will be presented to validate the method. Finally, Section 7 concludes this study.

\section{The Proposed Systematic Method}

A systematic method illustrated in Figure 1 is proposed to predict wind speed of wind farm, basically consisting of three parts: IGA-YanMeng typhoon numerical simulation model, typhoon status prediction model, and wind speed simulation based on extreme learning machine. The purpose of the first part is to generate a huge numerical wind speed training set using the YanMeng wind field according to some filtered typhoon data from the historical typhoon database provided by the Chinese meteorological station [16]. At the same time, multipoint measured data is used to enhance the simulation accuracy of the model by optimizing the key parameters through an improved genetic algorithm. The second part is the typhoon status prediction model, whose function is to predict the changes of typhoon parameters such as geographic coordinates, translation speed, translation direction, and so on in the future according to real-time information of the typhoon recorded in the previous moment. Then, the wind speed simulation model can obtain the wind speed magnitude/direction of each wind turbine based on an extreme learning machine through the predicted typhoon status from the second part. Finally, it is achievable to take measures before the accident happens to avoid or decrease the damage of the wind farm caused by the typhoon disaster.

\section{IGA-YanMeng Typhoon Numerical Simulation Model}

The basic function of the IGA-YanMeng typhoon numerical simulation model is to simulate the wind speed (including magnitude and direction) of each wind turbine under historical typhoons selected. These numerous wind speed data generated will be used as a huge training set to train the ELM later. This model can be mainly divided into four parts: data processing, IGA, historical typhoon filter, and YanMeng wind field, as shown in Figure 2.

The first step is to select available historical typhoons from the database provided by the local meteorological station using the filter. Figure 3 illustrates how it functions.

The filter calculates the distance between the center of the wind farm and each geographic position along the track of a historical typhoon. It is assumed that the historical typhoon influenced the wind farm if the distance is shorter than the scale of the typhoon, which is used to describe the size of different typhoons. For example, the center of the wind farm is within the influence range of typhoon 1 in Figure 3; therefore, typhoon 1 will be selected in the filter, while typhoon 2 will not. The geographical coordinates of the wind farm center can be calculated using the following equations:

$$
\begin{aligned}
L_{\mathrm{ltd}, c} & =\sum_{i=1}^{M_{t}} \frac{L_{\mathrm{ltd}, i}}{M_{t}}, \\
L_{\mathrm{lgd}, \mathrm{c}} & =\sum_{i=1}^{M_{t}} \frac{L_{\mathrm{lgd}, \mathrm{i}}}{M_{t}},
\end{aligned}
$$

where $L_{\mathrm{ltd}, c}$ is the latitude of the wind farm center, $L_{\mathrm{ltd}, i}$ is the latitude of the $i^{\text {th }}$ wind turbine in the wind farm, $L_{\operatorname{lgd}, c}$ is the longitude of the wind farm center, and $L_{\operatorname{lgd}, i}$ is the longitude of the $i^{\text {th }}$ wind turbine in the wind farm. $M_{t}$ is the total number of wind turbines in the wind farm.

Then, YanMeng wind field can simulate the $10 \mathrm{~min}$ average wind speed magnitude/direction at $10 \mathrm{~m}$ height of each wind turbine under the historical typhoons selected in the filter. YanMeng typhoon wind field considers the pressure gradient of the boundary layer friction, with equilibrium equations written as follows $[17,18]$ :

$$
\frac{\partial \mathbf{v}_{c}}{\partial t}+v_{c} \cdot \nabla \mathbf{v}_{c}=-\frac{1}{\rho_{a}} \nabla p_{a}-f_{c} \mathbf{k} \times \mathbf{v}_{c}+\mathbf{F}_{t},
$$

where $\mathbf{v}_{c}$ is the typhoon wind speed, $\rho_{a}$ is the air density, $f_{c}$ is the Coriolis force parameter, $\mathbf{F}_{t}$ is the boundary layer friction force, and $\mathbf{k}$ is a unit vector. The speed of air movement can be decomposed into the vector sum of the gradient wind speed, $\mathbf{v}_{g}$, and the surface friction wind speed $\mathbf{v}_{f}$ in the free boundary layer, expressed as follows:

$$
\mathbf{v}_{c}=\mathbf{v}_{f}+\mathbf{v}_{g} .
$$

The air pressure model used in YanMeng's typhoon wind field is the Holland pressure field model, in which the pressure of the location at $r \mathrm{~km}$ from the typhoon center $P(r)$ is [19]

$$
P(r)=P_{0}+\Delta P \cdot \exp \left[-\left(\frac{R_{m}}{r}\right)^{B}\right],
$$

where $P_{0}$ is the typhoon center pressure, $R_{m}$ is the maximum wind speed radius. $B$ is the Holland pressure profile 


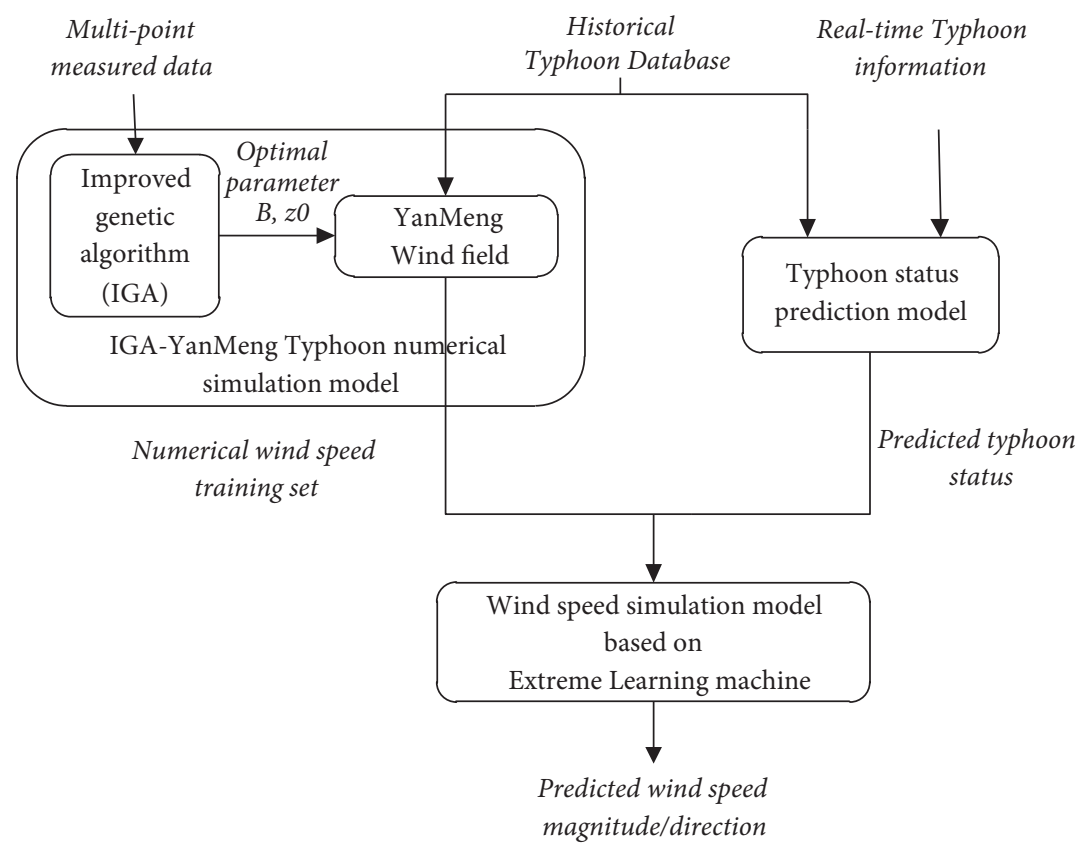

Figure 1: The flowchart of the systematic method.

parameter, and $\Delta P=P_{\infty}-P_{0}$ with $P_{\infty}$ taken as $1,010 \mathrm{hPa}$. In general, the maximum gradient wind speed radius can be approximated as the maximum wind speed radius, and the pressure gradient along the radial distribution can be described as follows:

$$
\frac{\partial P}{\partial r}=\frac{\Delta P B}{r}\left(\frac{R_{\max }}{r}\right)^{B} \exp \left[-\left(\frac{R_{\max }}{r}\right)^{B}\right]
$$

At the same time, some measured wind speed data of the wind farm will be used to enhance the simulation accuracy of YanMeng wind field through IGA. Figure 4 shows how IGA functions in this process in detail. Data processing is the first step. The measured wind speed data (magnitude and direction) will be recorded by the sensors set at multiple wind turbines among the farm. However, under such extreme typhoon conditions, some wind speed sensors may suffer damage, resulting in abnormal measured data such as data missing, abnormal data, and so on. Thence, these original data required necessary processing before further use. Figure 5 explains two approaches, Karman-window method and envelope filtering used in the data processing.

Firstly, the abnormal data point needs to be eliminated using Karman-window method. The basic idea is to predict the wind speed of the next moment and determine a range named detection window that is calculated according to the previous series of wind speed. For example, $y_{t-1}$ is the wind speed magnitude of the last moment; $y_{t}$ is the wind speed magnitude of current moment $t ; y_{k, t}$ is the predicted wind speed of the current moment obtained using the Karman filter; and $K_{C}$ is the scale factor that can control the size of window. $R_{\mathrm{inc}, t}$ and $R_{\mathrm{dec}, t}$ determine the range of the detection window. The data will be regarded as abnormal if it goes beyond the detection window. $R_{\text {inc, } t}$ is the increase limit range of the detection window at current moment $t$.
And $R_{\mathrm{dec}, t}$ is the decrease limit range of the detection window at current moment $t$. They are given by the following equations:

$$
\begin{aligned}
& R_{\text {inc }, t}=\frac{\left(\sum_{n=1}^{20} \Delta_{\text {inc }, t-n}\right)}{20}, \\
& R_{\text {dec }, t}=\frac{\left(\sum_{n=1}^{20} \Delta_{\mathrm{dec}, t-n}\right)}{20},
\end{aligned}
$$

where $\Delta_{\text {inc, } t-n}$ is the increment of wind speed at moment $t-n$ and $\Delta_{\mathrm{dec}, t-n}$ is the decrease of wind speed at moment $t-n$.

Then, the envelope filtering method is proposed to extract the average wind from the original wind data. As can be seen in Figure 5, the original wind speed data is very scattered because of the pulsating wind composition, which required elimination. The upper envelope line and lower envelope line that surround the original data can be determined by analyzing the turning points of the curve. Then the filtered wind speed data can be calculated using the following equation:

$$
y_{i, f}=\frac{\left(y_{i, u}+y_{i, l}\right)}{2}
$$

where $y_{i, f}$ is wind speed after filtering at moment $i, y_{i, u}$ is the wind speed on the upper envelope line at moment $i$, and $y_{i, l}$ is the wind speed on the lower envelope line at moment $i$.

As can be seen in Figure 4, the second step of IGA is selection. The available measured wind speed data of wind turbines will be put into comparison with those simulated data from YanMeng wind field. The error between the measured data and simulated data will be used to enhance the simulation accuracy by optimizing two important parameters, $B$ and $z_{0}$ of YanMeng wind field using IGA. 


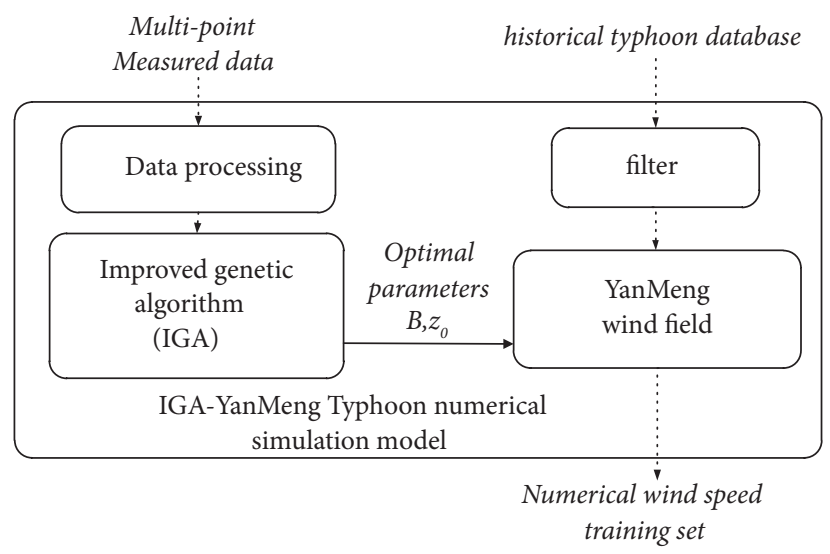

FIGURE 2: Flowchart of IGA-YanMeng typhoon numerical simulation model.

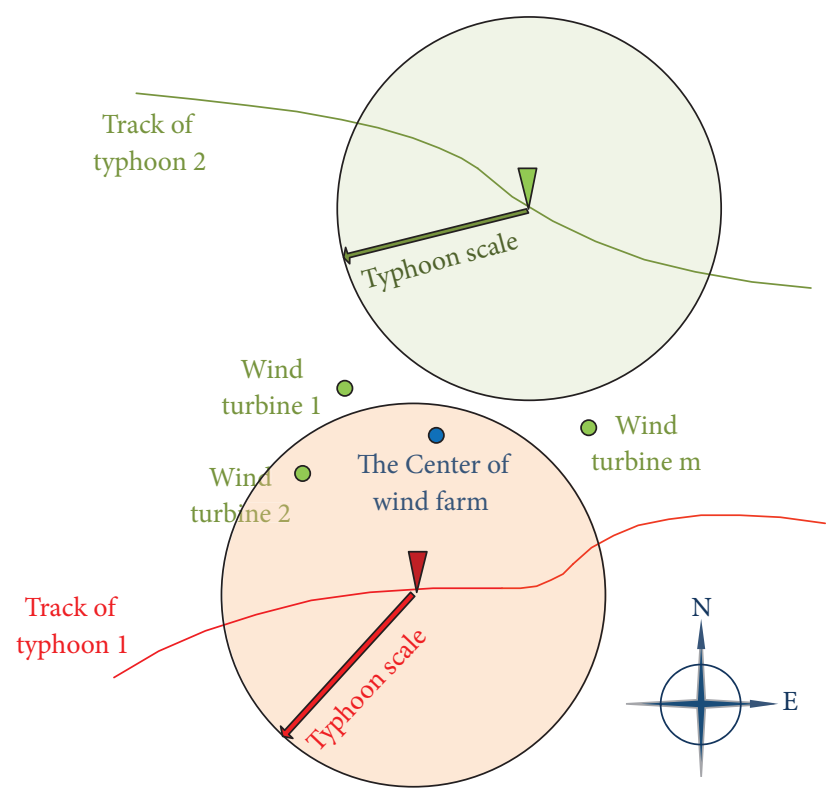

Figure 3: Historical typhoon filter.

The fitness value $F$ is used to evaluate the performance of parameters combination as follows:

$$
F=\frac{1}{\sqrt{\sum_{i}\left[\left(\left(v_{t, i}-v_{r, i}\right) / v_{r, i}\right)^{2}+\left(\left(\psi_{t, i}-\theta_{r, i}\right) / \theta_{r, i}\right)^{2}\right]}},
$$

where $F$ is the fitness of an individual, $v_{s, i}$ is the simulated average wind speed at $i^{\text {th }}$ monitoring point, $v_{r, i}$ is the realtime measured average wind speed at $i^{\text {th }}$ monitoring point, $\Psi_{s, i}$ is the simulated wind direction at $i^{\text {th }}$ monitoring point, and $\theta_{r, i}$ is the real-time measured wind direction at $i^{\text {th }}$ monitoring point.

Those parameter combinations with higher fitness values will be reserved to the next generation in the genetic algorithm.

$$
P_{x}=\frac{F_{x}}{\sum_{k=1}^{n} F_{k}}
$$

where $P_{x}$ is the probability of selection for parameter combination $x, F_{x}$ is the fitness value of parameter combination $x$, and $n$ is the population size, the number of individuals of a generation.

Then IGA will judge whether the simulation performance of YanMeng wind field model meets the accuracy conditions. If the result is negative, the process will go to crossover and mutation. Crossover and mutation in IGA can assure the diversity of the parameter population, which can help avoid local optimization. What is noticeable is that the directional mutation in IGA is different from the typical GA, which is totally random [20]. The mutation direction depends on the overall situation of simulation error of all points. The relative simulation error $e_{i}$ of point $i$ can be given by the following equation:

$$
e_{i}=\frac{\left(v_{t, i}-v_{r, i}\right)}{v_{r, i}}
$$




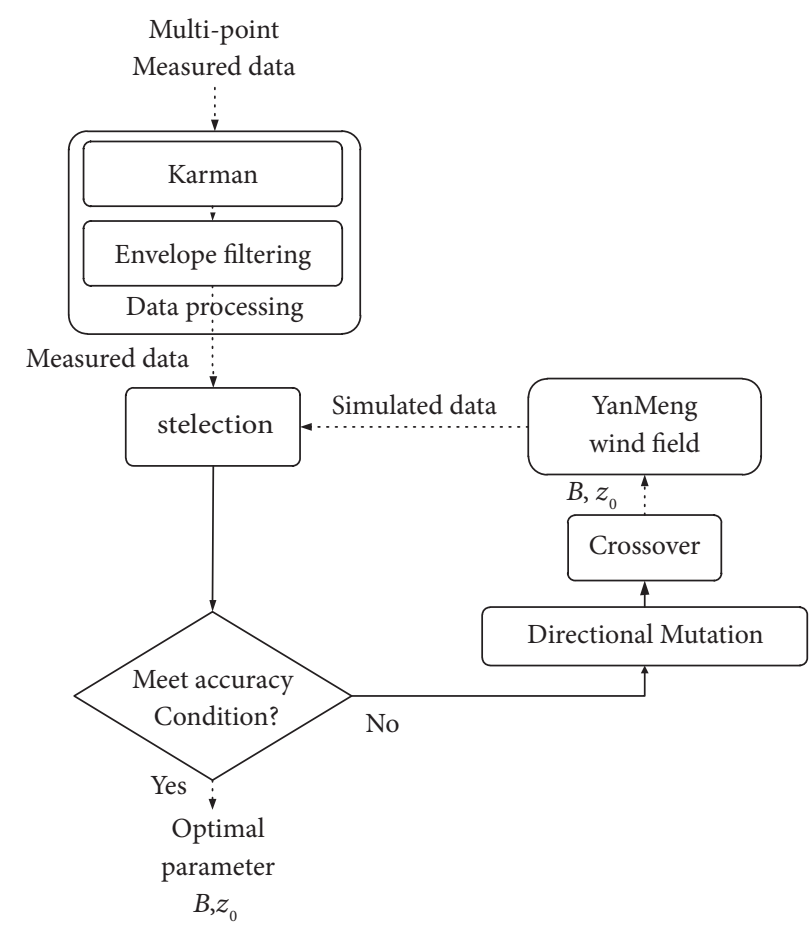

FIgURE 4: Flowchart of IGA.

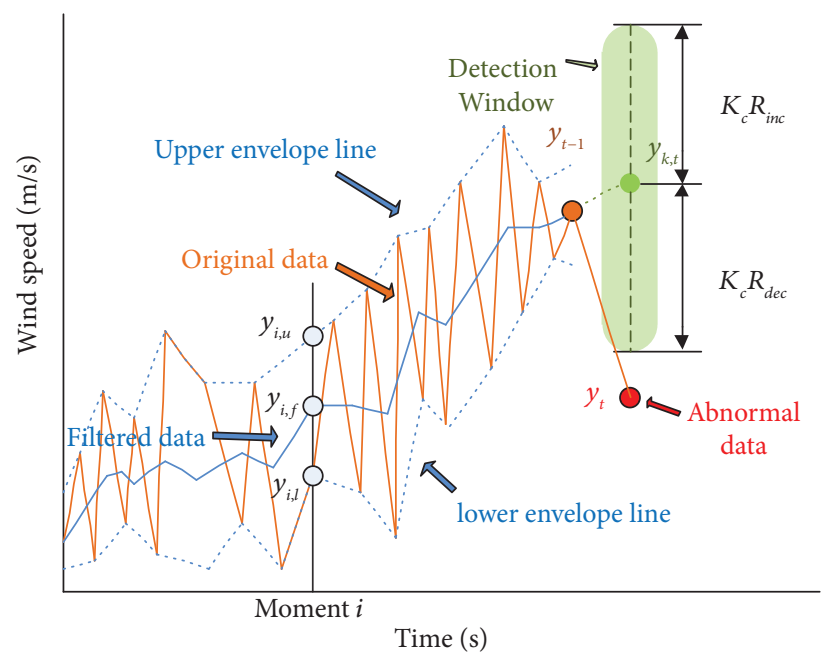

Figure 5: Data processing.

An error coefficient $E$ is used to describe the holistic error as follows:

$$
E=\frac{\sum_{i} e_{i}}{\sum_{i}\left|e_{i}\right|} .
$$

When the error $e_{i}$ is positive, it is representing that the simulated wind speed is larger than the real-time measured one. The mutation runs without any direction to ensure global optimization when the difference is small. But when the difference comes big with quantitative description shown by the following formula, the mutation will happen with the direction that decreases the difference. The direction depends on whether the error $e_{i}$ is positive or negative.

$$
\begin{array}{r}
E \geq 0.5 \\
\text { or } E \leq-0.5 .
\end{array}
$$

Parameters $B$ and $z_{0}$ after directional mutation and crossover will be imported into the YanMeng wind field for simulating data. The process will go back to selection for a new round of iteration. And it does not end until the accuracy conditions are met. Finally, the optimal parameters $B$ 
and $z_{0}$ will be used for generating a numerical wind speed training set, as can be seen in Figure 2.

\section{Typhoon Status Prediction Model}

The second part of the systematic method is the typhoon status prediction model, whose purpose is to predict the status of typhoons studied in the next few hours. The status includes six key parameters of the typhoon, as shown in Table 1 .

The model considers the historical typhoon data based on similarity and also the previous status of the typhoon itself. The main flowchart is shown in Figure 6.

Firstly, the season similarity filter checks the occurrence month of the historical typhoons. The typhoon data will be eliminated if the time difference between the historical typhoon and the current one is more than a month. Then, an influence circle with a radius of $250 \mathrm{~km}$ as shown in Figure 7 is used to select those historical typhoons closed to the position of the current typhoon.

In Figure 7, $p_{t}$ is the position of the current typhoon at the moment $t . p_{t+i}$ is the position of the current typhoon in the next $i$ hour. $p_{t-i}$ is the position of the current typhoon $i$ hour ago.

The similarity of the historical typhoon is given as follows:

$$
S_{g, m}=1-\left(\frac{d_{m}}{R}\right)^{2}
$$

where $S_{g, m}$ is the geographic similarity of $p_{m}$ on the historical typhoon track, $d_{m}$ is the distance between $p_{m}$ and $p_{t}$ in $\mathrm{km}$, and $R$ is the radius of the influence circle; it is taken as $250 \mathrm{~km}$.

$$
S_{v, m}=1-\left|\frac{\left(v_{m}-v_{t}\right)}{v_{t}}\right|^{2}
$$

where $S_{y, m}$ is the translation speed similarity of $p_{m}, v_{m}$ is the translation speed of $p_{m}$ in $\mathrm{km} / \mathrm{h}$, and $v_{t}$ is the translation speed of $p_{t}$ in $\mathrm{km} / \mathrm{h}$.

$$
S_{\theta, m}=1-\left|\frac{\left(\theta_{m}-\theta_{t}\right)}{\theta_{t}}\right|^{2}
$$

where $S_{\theta, m}$ is the translation direction similarity of $p_{m}, \theta_{m}$ is the translation direction of $p_{m}$, and $\theta_{t}$ is the translation speed of $p_{t}$.

The overall similarity of $p_{m}$ is as follows:r

$$
S_{m}=\sqrt{S_{g, m}^{2}+S_{v, m}^{2}+S_{\theta, m}^{2}} .
$$

The correlation between previous status and future one of current typhoon is also taken into consideration. It is given by the following equation:

$$
C_{t-i}=1-\left(\frac{d_{t-i}}{R}\right)^{2}
$$

where $C_{t-i}$ is the correlation value of $p_{t-i}$ on the previous track of the current typhoon. $d_{t-i}$ is the distance between $p_{t}$ and $p_{t-i}$ in $\mathrm{km}$.

In this model, the status of the current typhoon is described by two vectors. One is parameter set $\lambda_{t}$, including six key parameters mentioned in Table 1:

$$
\lambda_{t}=\left[V_{\max , t}, p_{\text {air }, t}, v_{t}, \theta_{t}, \varphi_{\mathrm{ltd}, t}, \varphi_{\mathrm{lgd}, t}\right],
$$

where $V_{\max , t}$ is the maximum wind speed among the typhoon structure at moment $t$ in $\mathrm{km} / \mathrm{h}, p_{\mathrm{air}, t}$ is air pressure of the typhoon center at moment $t$ in $\mathrm{hPa}, v_{t}$ is translation speed of typhoon center at moment $t$ in $\mathrm{km} / \mathrm{h}, \theta_{t}$ is translation direction of typhoon center at moment $t$ in ${ }^{\circ}, \varphi_{\mathrm{ltd}, t}$ is the latitude of typhoon center at moment $t$ in ${ }^{\circ}$, and $\varphi_{\operatorname{lgd}, t}$ is the longitude of typhoon center at moment $t$ in ${ }^{\circ}$.

The other is parameter change rate set $\Delta \lambda$, which is expressed as follows:

$$
\Delta \lambda_{t}=\left[\Delta V_{\max , t}, \Delta p_{\text {air }, t}, \Delta v_{t}, \Delta \theta_{t}, \Delta \varphi_{\text {ltd }, t}, \Delta \varphi_{\operatorname{lgd}, t}\right],
$$

where $\Delta \lambda_{t}$ is the 1 hour rate of change of set $\lambda_{t}$, and it is used to predict typhoon status in the future. $\Delta \lambda_{t}$ is the weighted value of the parameter change rate set for all points in the influence circle, which can be calculated using the following equation:

$$
\Delta \lambda_{t}(i)=\frac{\left(\sum_{m}^{M_{h}} \Delta \lambda_{m}(i) S_{m}+\sum_{n}^{M_{c}} \Delta \lambda_{n}(i) C_{n}\right)}{H}, \quad i=1,2, \ldots, 6,
$$

$$
H=\left(\sum_{m}^{M} S_{m}+\sum_{n}^{N} C_{n}\right)
$$

where $\Delta \lambda_{t}(i)$ is the No. $i$ element of set $\Delta \lambda_{t}, M_{h}$ is the number of historical typhoon points in the influence circle, and $M_{c}$ is the number of current typhoon points in the influence circle.

Finally, the typhoon status of the next hour described with $\lambda_{t+1}$ can be predicted by the following equation:

$$
\lambda_{t+1}=\lambda_{t}+\Delta \lambda_{t}
$$

\section{Wind Speed Simulation Model Based on ELM}

This model simulates the average wind speed magnitude/ direction at $10 \mathrm{~m}$ height of each turbine in the farm according to the predicted status. Before the simulation, the huge numerical wind speed training set will be used to train the ELM, making it more accurate.

The basic structure of ELM is shown in Figure 8.

The input data can be written as a column vector $\mathrm{X}$ as follows:

$$
X=\left[\begin{array}{c}
x_{1} \\
x_{2} \\
\vdots \\
x_{m}
\end{array}\right]_{M \times 1},
$$


TABLE 1: Key parameters of the typhoon.

\begin{tabular}{lc}
\hline Parameter & Description of parameter \\
\hline$V_{\max }$ & The maximum wind speed among the typhoon structure in $\mathrm{m} / \mathrm{s}$ \\
$p_{\text {air }}$ & Air pressure of the typhoon center in $\mathrm{hPa}$ \\
$v_{c}$ & Translation speed of typhoon center in $\mathrm{km} / \mathrm{h}$ \\
$\theta$ & Translation direction of typhoon center in ${ }^{\circ}$ \\
$\varphi_{\text {ltd }}$ & Latitude of typhoon center in ${ }^{\circ}$ \\
$\varphi_{\mathrm{lgd}}$ & Longitude of typhoon center in
\end{tabular}

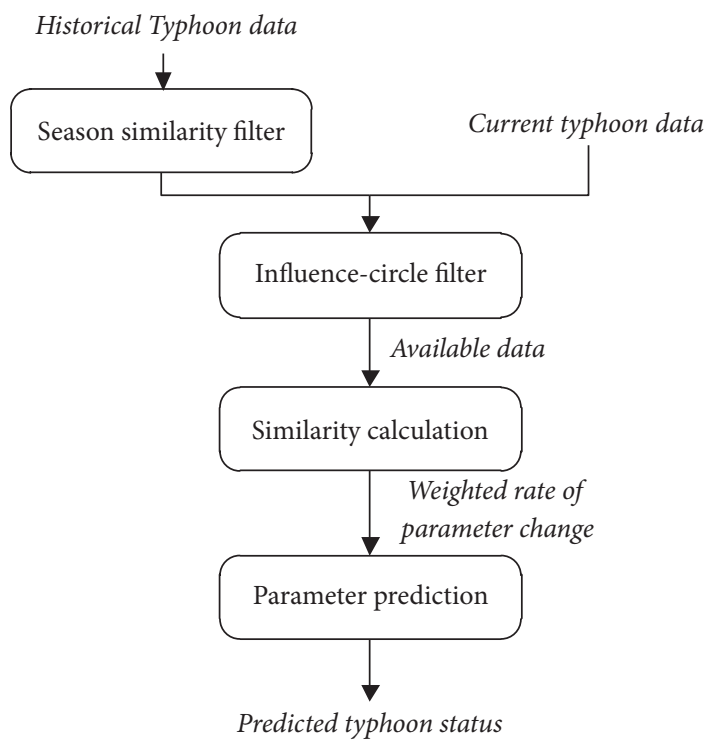

FIGURE 6: Flowchart of typhoon status prediction model.

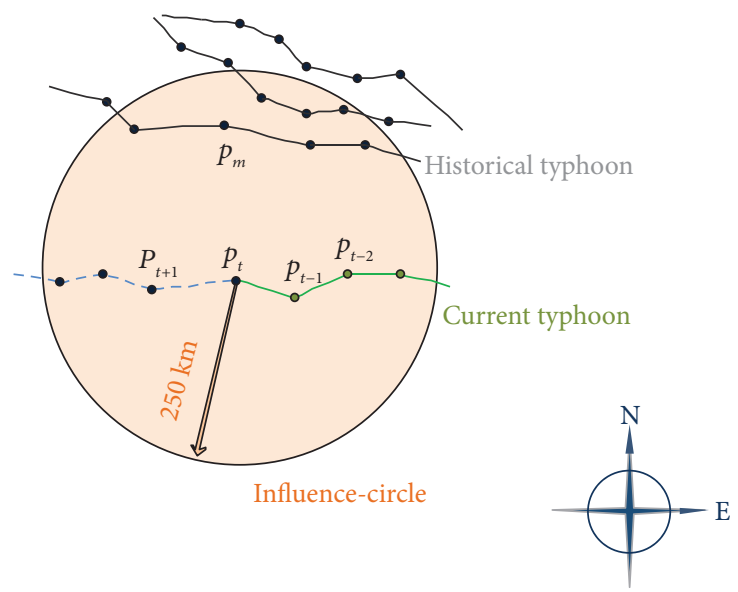

FIgURE 7: Influence circle.

where $x_{m}$ is the input data type $m$ and $M$ is the number of data types.

The second layer, hidden layer consists of a certain number of hidden neurons. Each neuron with three attributes, input weight, bias, and output weight, stands for the correlation between the input layer and the output layer.

The input weight matrix $W$ is written as follows [21]:

$$
W=\left[\begin{array}{cccc}
w_{1,1} & w_{1,2} & \cdots & w_{1, N} \\
w_{2,1} & w_{2,2} & \cdots & w_{2, N} \\
\vdots & w_{m, n} & \vdots \\
w_{M, 1} & w_{M, 2} & \cdots & w_{M, N}
\end{array}\right]_{M \times N} .
$$

The bias column vector $B$ is written as follows [21]: 


$$
B=\left[\begin{array}{c}
b_{1} \\
b_{2} \\
\vdots \\
b_{n}
\end{array}\right]_{N \times 1}
$$
[21]:

And the output weight matrix $Q$ is written as follows

$$
Q=\left[\begin{array}{cccc}
q_{1,1} & q_{1,2} & \cdots & q_{1, K} \\
q_{2,1} & q_{2,2} & \cdots & q_{2, K} \\
& \vdots & q_{n, k} & \vdots \\
q_{N, 1} & q_{N, 2} & \cdots & q_{N, K}
\end{array}\right]_{N \times K}
$$

where $N$ is the number of neurons in the hidden layer and $K$ is the number of the output data types; there are two output data types, wind speed magnitude and wind speed direction in the research, and the $K$ is therefore taken as 2. $w_{m, n}$ represents the input weight value of input data type $m$ and the neuron $n . b_{n}$ represents the bias value of neuron $n$ and $q_{n, k}$ represents the output weight value of neuron $n$ and the output data $k$.

The relation between output $Y$ and input $X$ can be described as follows [21]:

$$
y_{k}=\sum_{n=1}^{N} q_{n, k} g\left(\sum_{m=1}^{M} w_{m, n} x_{m}+b_{n}\right), \quad k=1,2, \ldots, K,
$$

where $g(x)$ is an activation function and $y_{k}$ is the output data type $k$.

The wind speed simulation model is like a mapping from typhoon information to the average wind speed of a certain turbine position. In other words, more valuable information is necessary to ensure the performance of the ELM network. Therefore, other kinds of information of typhoon such as the distance between the target point and typhoon center as well as the azimuth of the target point among the typhoon structure will be added to the input data types.

In a typical ELM, the input weight matrix $W$ and the bias column vector are generated randomly. The essence of solving the ELM network is to solve the output weight matrix $Q$ through the training set [21]. In this study, the ELM is used to simulate the average wind speed data of each turbine position according to the corresponding key parameters of the typhoon. The situation is that not every single parameter is beneficial to the simulation accuracy because the correlation between wind speed data and different parameters is not the same. Therefore, PSO will be used to optimize the input weight matrix $W$ and the bias column vector $B$ of the ELM network.

PSO algorithm is the optimization method inspired by the birds flocking phenomenon in nature [22]. It has been widely used for its convenience and efficiency. Imagine there is a huge flock of birds searching for food in a forest. Every single bird moves in different locations in the forest. In this process, a bird learns where to find more food based on its own searching experience, which is called individual experience in PSO. At the same time, it communicates with other birds about the best place, which is called social experience. Then the birds adjust their searching strategy to get more food. This is exactly how the PSO algorithm works.

The basic flowchart of PSO is shown in Figure 9.

In the swarm initialization section, a swarm of 500 birds are generated based on the Pearson correlation analysis. The initial value will be determined according to the corresponding correlation between different data types in the ELM network. Each bird has a scheme of parameters $W$ and $B$ [22]:

$$
P_{\text {ind }}=\left[p_{\text {ind }, 1}, p_{\text {ind }, 2}, \ldots, p_{\text {ind }, D}\right],
$$

where $P_{\text {ind }}$ is the individual parameter scheme of a bird and $D$ is the number of elements in matrix $W$ and $B$.

Then, every bird's performance is judged in the fitness calculation section using equation (30) [22]. And a bird will update the best scheme of its own based on searching experience, which is known as individual experience in PSO. At the same time, the social experience, the best scheme of the whole bird swarm will also be refreshed.

$$
F_{\mathrm{PSO}}=\frac{1}{\sqrt{\left(e_{v}^{2}+e_{d}^{2}\right)}}
$$

where $e_{v}$ is the mean squared error (MSE) of wind speed magnitude simulation with ELM and $e_{d}$ is the MSE of wind speed direction simulation with ELM.

$$
\begin{aligned}
G_{\text {ind }} & =\left[g_{\text {ind }, 1}, g_{\text {ind }, 2}, \ldots, g_{\text {ind }, D}\right], \\
G_{\text {swm }} & =\left[g_{\mathrm{swm}, 1}, g_{\mathrm{swm}, 2}, \ldots, g_{\mathrm{swm}, D}\right],
\end{aligned}
$$

where $G_{\text {ind }}$ represents the best scheme of an individual bird based on its own searching experience. And $G_{\text {swm }}$ represents the best scheme of the whole bird swarm.

Finally, each bird adjusts their searching strategy and moves to a new location to improve its own performance according to individual experience and social experience. The adjustment is described with $V_{\text {ind }}$ [22] as follows:

$$
\begin{aligned}
V_{\text {ind }}= & c_{\text {ita }} V_{\text {ind,last }}+c_{\text {ind }} r_{\text {ind }}\left(G_{\text {ind }}-P_{\text {ind }}\right) \\
& +c_{\text {swm }} r_{\text {swm }}\left(G_{\text {swm }}-P_{\text {ind }}\right),
\end{aligned}
$$

where $V_{\text {ind }}$ is the adjustment of $P_{\text {ind }}$ and $V_{\text {ind, last }}$ is the adjustment of $P_{\text {ind }}$ for the last iteration. $c_{\text {ita }}$ is the inertia constant, $c_{\text {ind }}$ is the constant of individual experience, $c_{\text {swm }}$ is the constant of social experience, $r_{\text {ind }}$ is the random value for individual experience in the interval $(0,1)$, and $r_{\text {swm }}$ is the random value for social experience in the interval $(0,1)$.

$$
P_{\text {ind }}^{\prime}=P_{\text {ind }}+V_{\text {ind }} \text {, }
$$

where $P_{\text {ind }}^{\prime}$ is the new individual parameter scheme of a bird.

\section{Case Study}

This section presents a case study on a wind farm located in Guangdong province suffering severe damage when the super typhoon Mangkhut landed in 2018. The systematic 


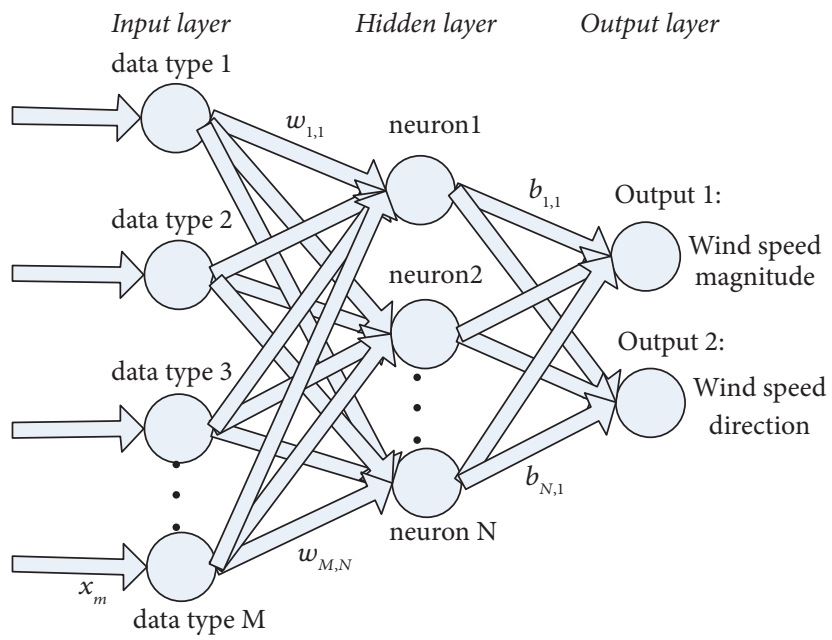

Figure 8: The structure of ELM.

method proposed in this paper is used to predict the average wind speed magnitude and direction of the turbine under the typhoon condition.

As the No. 22 typhoon in 2018, Mangkhut became a tropical storm in the west Pacific at 5:00 on September 8, 2018. Then it strengthened continuously and marched northwest toward China's southern sea. It finally developed into a super typhoon and landed at 17:00 on September 16 in Taishan city, Guangdong province [16].

In the first part of the systematic method, the IGAYanMeng typhoon numerical simulation model, a huge training set of typhoon parameter-turbine wind speed is generated through the YanMeng wind field according to the historical typhoon data containing information of 2,354 typhoons that landed on the Chinese coastline from 1949 to 2018 provided by China meteorological station [16]. At the same time, multipoint measured wind speed data covering $1,263,613$ moments during typhoon weather and 13 wind turbines positions in recent years are used to improve the accuracy of the training set, optimizing the parameters $B$ and $z_{0}$ in YanMeng wind field.

Meanwhile, the typhoon status prediction model is used to forecast the typhoon parameter change in the next 1-6 hours based on historical typhoon similarity and Mangkhut's previous status correlation. In the process of the prediction for 1-6 hours, the prediction of typhoon parameter change is conducted hour by hour. For example, the model first predicts parameter change in the next 1 hour. Then on this basis, the model will add the predicted status in the next $1 \mathrm{~h}$ to the typhoon previous status sequence and predict parameter change in the next $2 \mathrm{~h}$. And so on, the prediction of parameter change in the next $1-6 \mathrm{~h}$ comes out. Figure 9 presents the result of Mangkhut's status prediction at 13:00 on September 16. The actual track is marked with white arrows and white lines. The six predicted positions of Mangkhut from $1 \mathrm{~h}$ to $6 \mathrm{~h}$ ahead of 13:00 are marked with yellow dots.

As can be seen in Figure 10, in the $4 \mathrm{~h}$ ahead of 13:00, the predicted positions are rather closed to the actual ones. However, when it comes to $5 \mathrm{~h}$ to $6 \mathrm{~h}$ ahead, it is quite difficult for the model to predict the actual trend and the error goes big.

Particular focus is placed on the prediction performance from 9:00 on September 16, 2018, to 6:00 on September 17, 2018, when Mangkhut landed onshore and swept across Guangdong province, to better validate the systematic method as a whole. Table 2 shows the root-mean-squared error (RMSE) of prediction for each typhoon key parameter $1 \mathrm{~h}$ ahead to $6 \mathrm{~h}$ ahead during the period, while Figure 11 shows the curve of predicted parameters.

It can be seen from Table 2 that the RMSE of key parameter prediction $1 \mathrm{~h}$ ahead is the smallest, while the RMSE of $6 \mathrm{~h}$ ahead is the largest, which infers that when the prediction time interval gets bigger, the RMSE grows bigger correspondingly.

Then the wind speed simulation model generates the average wind speed at $10 \mathrm{~m}$ height including the magnitude and direction of every single turbine in the wind farm according to the predicted typhoon key parameters. Before that, the huge training set of typhoon key parameter-turbine wind speed is used to train the ELM network with PSO, making it more accurate. Figure 12 shows the simulation performance of ELM with PSO.

It can be seen in Figure 12 that the RMSE converged to the minimum of 0.3428 at epoch 860 for wind speed simulation. As for wind direction simulation, the RMSE converged to the minimum of 1.1251 at epoch 289 .

Table 3 shows the RMSE value and the R index of ELM with PSO.

RMSE stands for the simulation error, and the R index represents the correlation between the input and the output of ELM. It can be seen from Table 3 that the RMSE of the validation set came to 0.342837 for wind speed simulation, and it came to 1.125069 for wind direction simulation. $\mathrm{R}$ index of validation set reached 0.998912 for wind speed simulation, and it reached 0.999741 for wind direction simulation. It can be inferred that the wind speed simulation model based on ELM shows extraordinary performance.

Figure 13 shows the wind speed magnitude/direction prediction results of wind turbine No. 13 with coordinates 


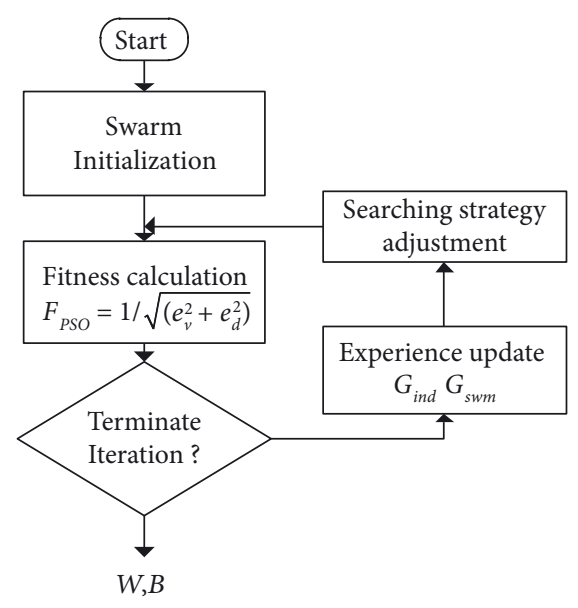

FIGURE 9: Flowchart of PSO.

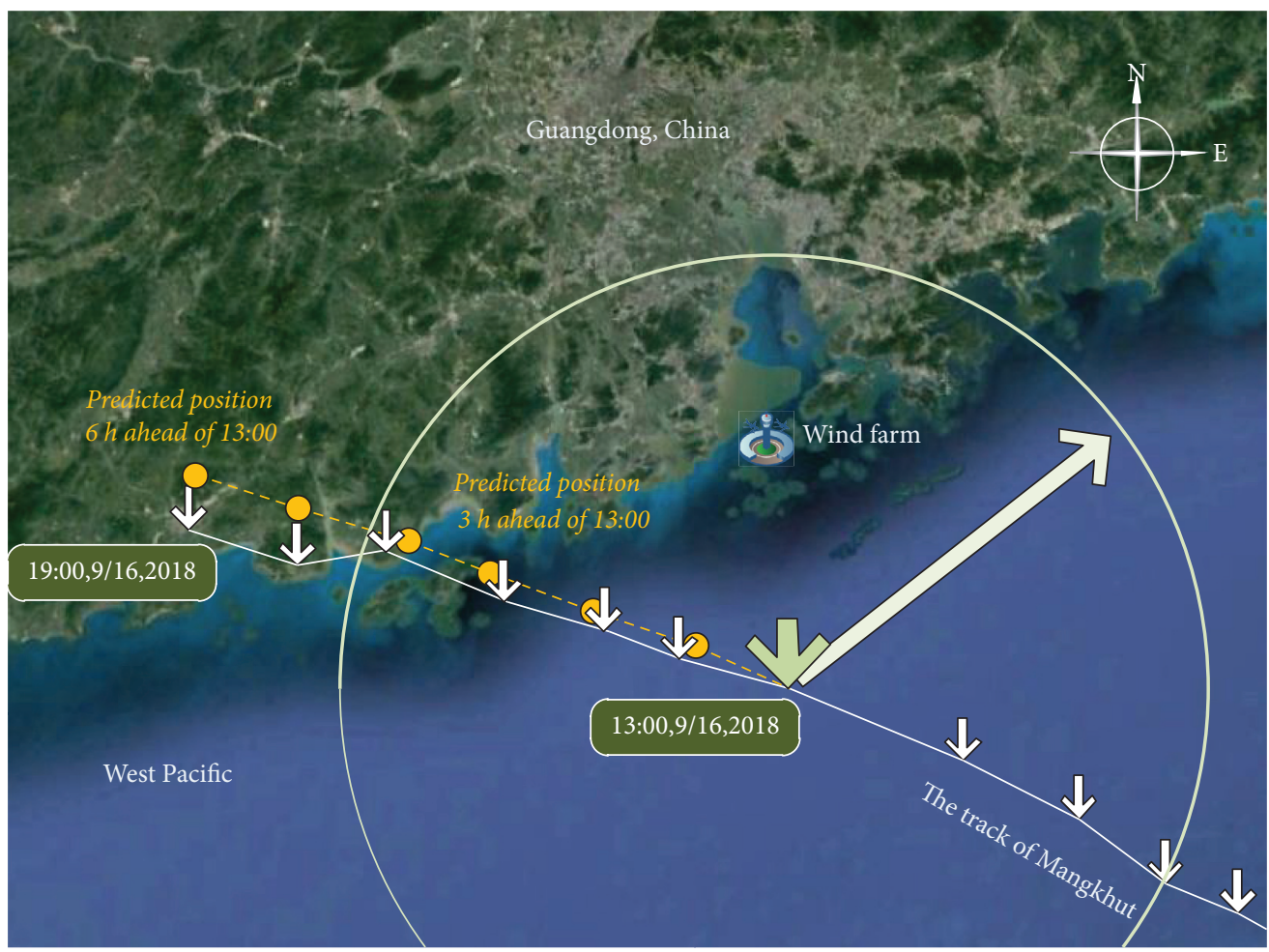

Typhoon position predicted at 13:00,9/16,2018

ל Typhoon actual position

FIGURE 10: Mangkhut's predicted status in $6 \mathrm{~h}$ at 13:00 on September 16, 2018.

TABLE 2: The overall RMSE of typhoon key parameter prediction.

\begin{tabular}{|c|c|c|c|c|c|c|}
\hline Key parameter & $1 \mathrm{~h}$ ahead & $2 \mathrm{~h}$ ahead & $3 \mathrm{~h}$ ahead & $4 \mathrm{~h}$ ahead & $5 \mathrm{~h}$ ahead & $6 \mathrm{~h}$ ahead \\
\hline$V_{\max }$ & 1.730064 & 2.563183 & 3.569731 & 4.43167 & 5.274586 & 5.973571 \\
\hline$P_{\text {air }}$ & 2.715035 & 3.783636 & 5.132059 & 6.172818 & 7.148655 & 8.036933 \\
\hline$v_{c}$ & 2.468804 & 2.990488 & 3.19536 & 3.716567 & 4.315859 & 4.417815 \\
\hline$\theta$ & 19.92125 & 20.02723 & 21.53194 & 23.19538 & 28.60333 & 30.37751 \\
\hline$\varphi_{\text {ltd }}$ & 0.11604 & 0.18525 & 0.239314 & 0.302295 & 0.384715 & 0.4521 \\
\hline$\varphi_{\text {lgd }}$ & 0.072334 & 0.106627 & 0.143203 & 0.180261 & 0.231382 & 0.293897 \\
\hline
\end{tabular}

Note: from 9:00, September 16, 2018, to 6:00, September 17, 2018. 


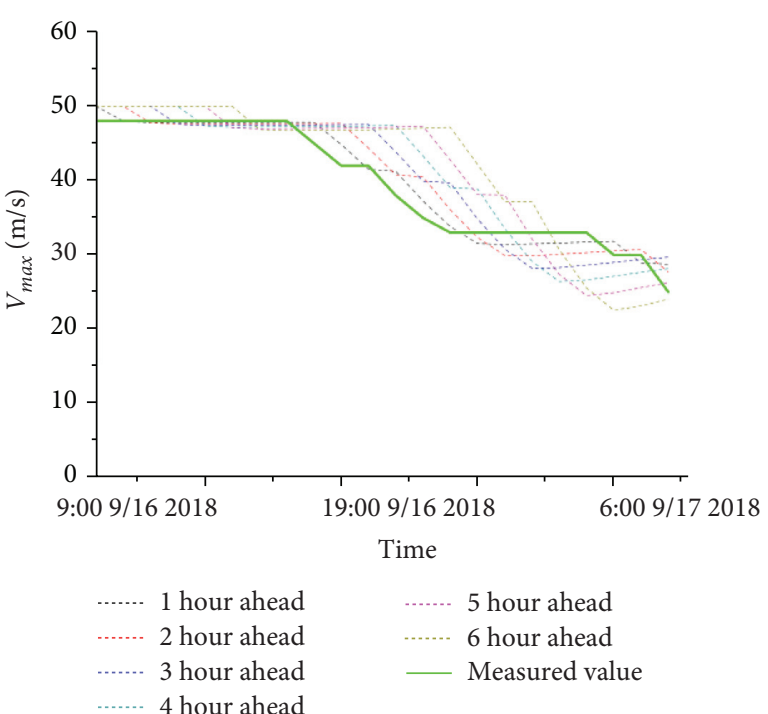

(a)

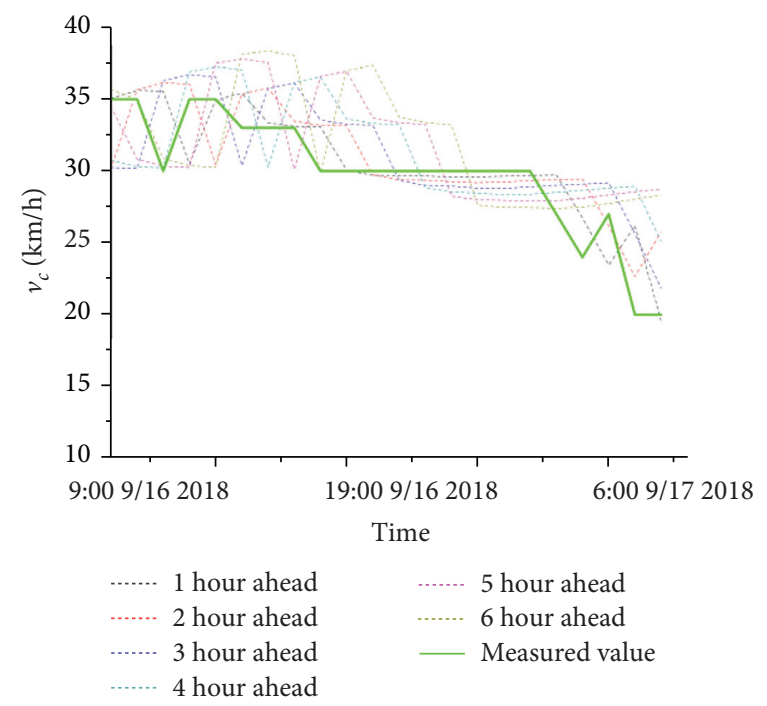

(c)

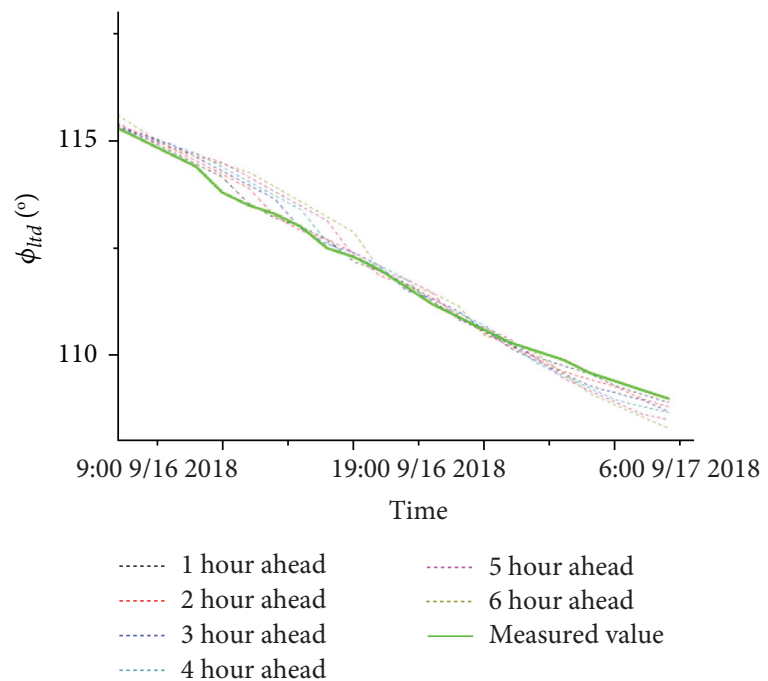

(e)

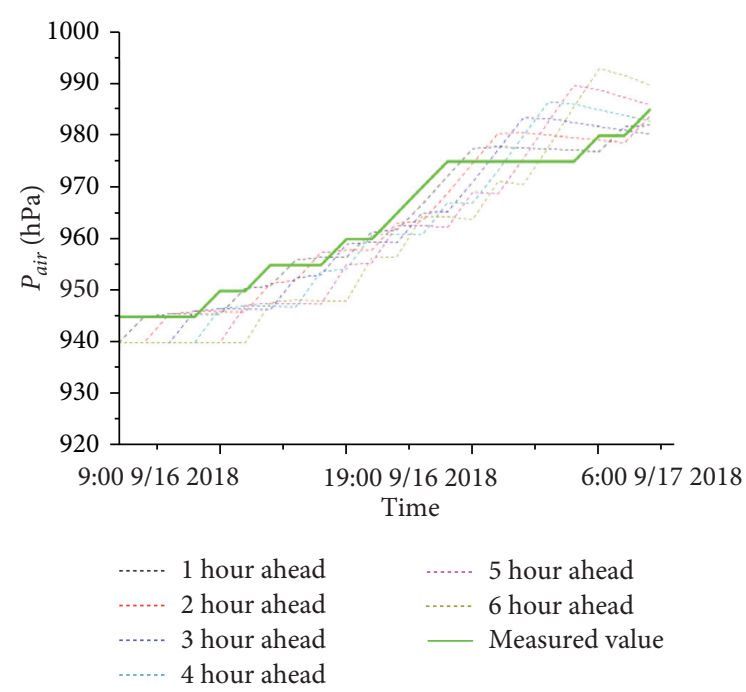

(b)

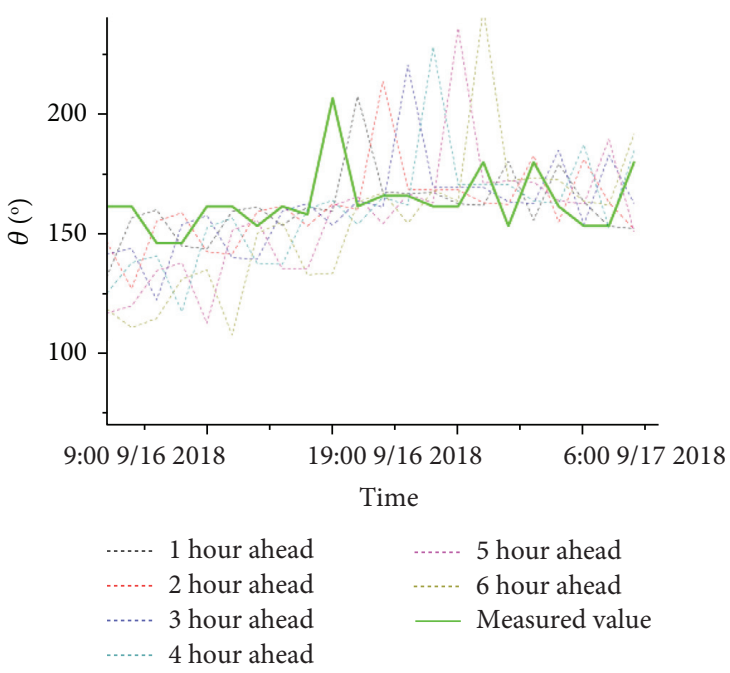

(d)

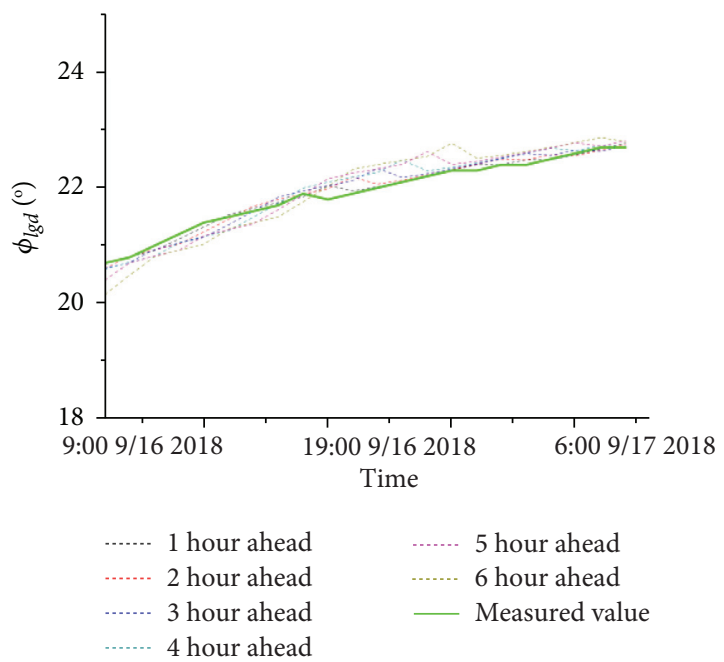

(f)

FIgURE 11: Prediction of typhoon parameters: (a) the maximum wind speed among the typhoon structure $V_{\max }$, (b) air pressure of the typhoon center $p_{\text {air }},(\mathrm{c})$ translation speed of typhoon center $v_{c}$, (d) translation direction of typhoon center $\theta$, (e) latitude of typhoon center $\varphi_{\text {ltd }}$, and (f) longitude of typhoon center $\varphi_{\text {lgd }}$. 


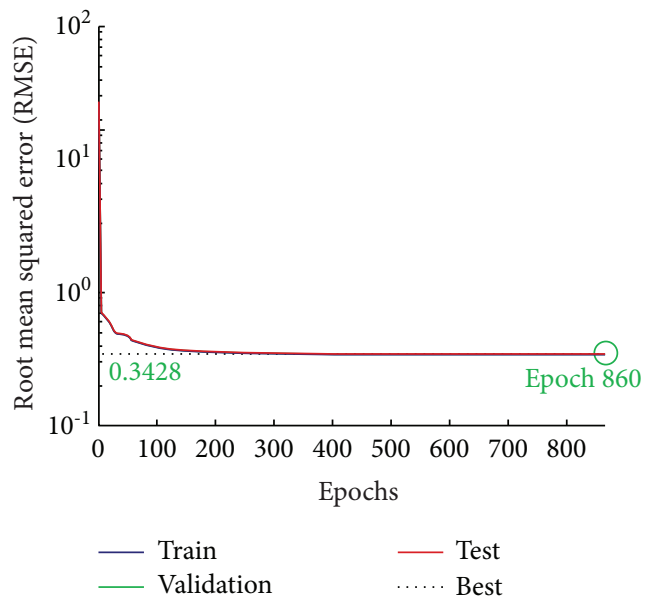

(a)

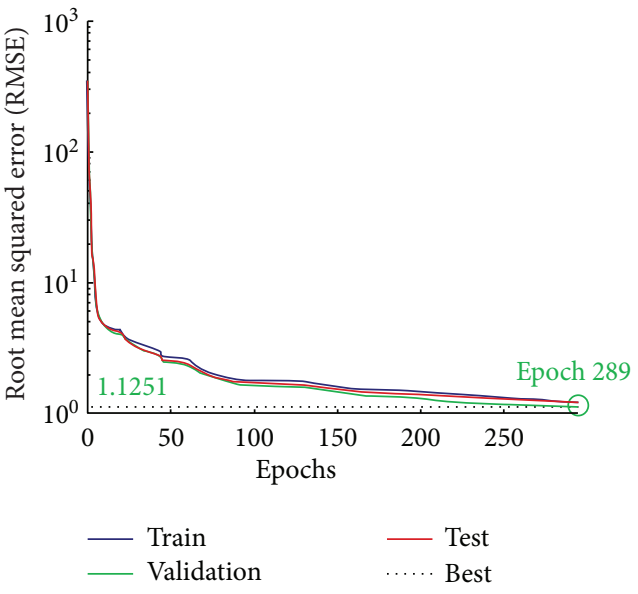

(b)

FIGURE 12: The performance of ELM with PSO: (a) wind speed simulation and (b) wind direction simulation.

TABLE 3: RMSE and R index of ELM with PSO.

\begin{tabular}{lcccr}
\hline & & Wind speed & \multicolumn{2}{c}{ Wind direction } \\
& RMSE & R & RMSE & 0.999692 \\
\hline Training set & 0.344394 & 0.998910 & 1.209380 & 0.999741 \\
Validation set & 0.342837 & 0.998912 & 1.125069 & 0.999691 \\
Test set & 0.343006 & 0.998906 & 1.219426 & \\
\hline
\end{tabular}

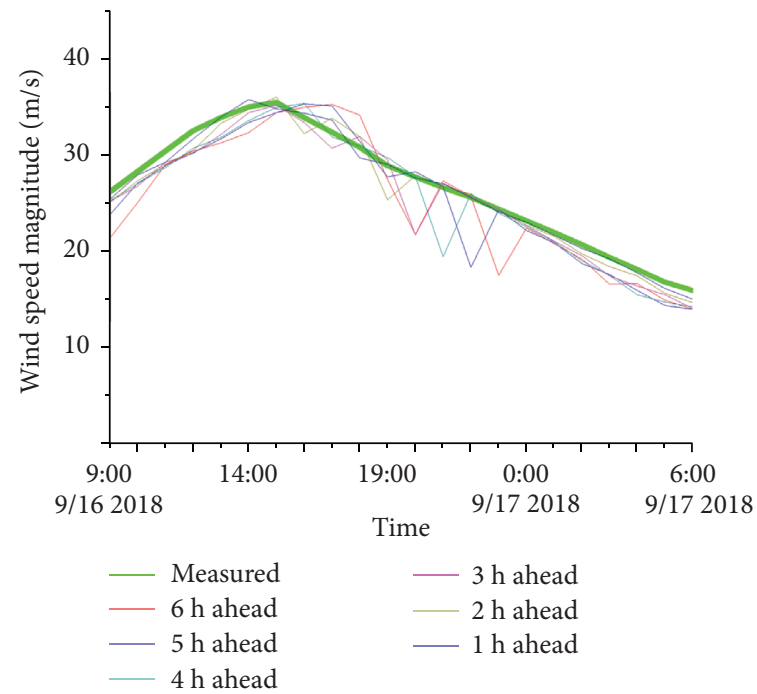

(a)

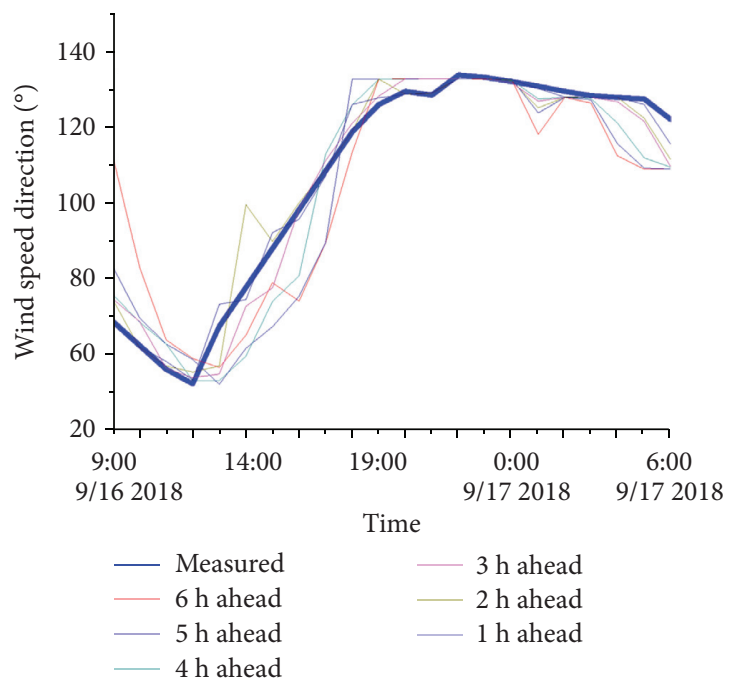

(b)

FIGURE 13: Wind speed prediction results of wind turbine No. 13: (a) wind speed magnitude and (b) wind speed direction.

$\left(113.728^{\circ} \mathrm{E}, 22.137^{\circ} \mathrm{N}\right)$ from 9:00 on September 16, 2018, to 6: 00 on September 17, 2018.

In Figure 13, the bold green curve represents the measured wind speed magnitude. The bold blue curve represents the measured wind speed direction. And the other thin curves stand for the predicted one. It can be seen that the curves of $1 \mathrm{~h}$ ahead to $3 \mathrm{~h}$ ahead are quite close to that of measured data, whether it is for magnitude or direction. However, the curves of $4 \mathrm{~h}$ ahead to $6 \mathrm{~h}$ ahead are more unstable in some parts. And there are larger differences between them and the measured data. The detailed error information is given by Table 4 .

For the wind speed magnitude, prediction $1 \mathrm{~h}$ ahead is the most accurate with the RMSE of 0.625876. The RMSE 
TABLE 4: RMSE of wind speed prediction of wind turbine No. 13.

\begin{tabular}{lcccccc}
\hline & $1 \mathrm{~h}$ ahead & $2 \mathrm{~h}$ ahead & $3 \mathrm{~h}$ ahead & $4 \mathrm{~h}$ ahead & $5 \mathrm{~h}$ ahead & $6 \mathrm{~h}$ ahead \\
\hline Wind speed magnitude & 0.625876 & 1.242866 & 1.774625 & 2.050791 & 2.248948 & 2.882477 \\
Wind speed direction & 2.85382 & 6.236313 & 5.338348 & 8.972893 & 11.91879 & 14.68233 \\
\hline
\end{tabular}

Note: from 9:00, September 16, 2018, to 6:00, September 17, 2018.

gets bigger when the prediction time interval increases. And it reaches 2.882477 for that $6 \mathrm{~h}$ ahead. A similar pattern appears when it comes to the wind speed direction part. Among the $1 \mathrm{~h}$ prediction data, the smallest RMSE is 2.85382, while the largest one is 14.68233 . One interesting thing is that the RMSE of $3 \mathrm{~h}$ ahead is slightly smaller than that of $2 \mathrm{~h}$ ahead, which might be due to the unstable prediction in the long time period, especially when the typhoon track gets complicated in mountainous areas. However, the RMSE of direction prediction gets bigger when the prediction time interval increases from the whole.

\section{Conclusions}

(1) This paper proposed a systematic method to predict the average wind speed at $10 \mathrm{~m}$ height (including magnitude and direction) of the wind turbine. The method mainly contains three parts: the IGA-YanMeng typhoon numerical simulation model, typhoon status prediction model, and the wind speed simulation model. The first step is to generate a huge training set of typhoon key parameter, turbine wind speed. Meanwhile, the status of the typhoon is predicted based on the similarity of historical typhoons and the correlation of the current typhoon's previous status. Finally, the wind speed is predicted according to the predicted typhoon status with the ELM trained by the training set in the first step.

(2) This method innovatively uses the YanMeng wind field to enrich the training set of ELM to a great extent, which helps improve the accuracy of ELM. And in this process, a new approach named envelop filter is used to extract the average wind speed from the original wind data. What is more, two parameters, $B$ and $z_{0}$ in the YanMeng wind field, are optimized using IGA based on multipoint measured data in recent years.

(3) In the wind speed simulation model, PSO is used to determine the input weight matrix $W$ and bias column vector $B$ of the ELM network, making it more accurate when it simulates the wind speed of turbines. The RMSE of the validation set came to 0.342837 for wind speed simulation, and it came to 1.125069 for wind direction simulation. The $\mathrm{R}$ index of the validation set reached 0.998912 for wind speed simulation, and it reached 0.999741 for wind direction simulation.

(4) The end of the paper presents the prediction results of turbine No. 13 from 9:00 on September 16, 2018, to $6: 00$ on September 17, 2018, when the typhoon Mangkhut swept over. Overall, the RMSE of prediction $1 \mathrm{~h}$ ahead is the smallest. When the prediction time interval increases, RMSE becomes larger correspondingly, whether it is for magnitude or direction of wind speed. For the magnitude, the smallest RMSE is 0.625876 for $1 \mathrm{~h}$ ahead, while the largest is 2.882477 for $6 \mathrm{~h}$ ahead. And for the direction, the smallest RMSE is 2.85382 for $1 \mathrm{~h}$ ahead, while the largest is 14.68233 for $6 \mathrm{~h}$ ahead.

(5) It is feasible to judge the probability of wind speed exceeding the capacity of the wind turbine in the next few hours according to the prediction results of the systematic method. And take some effective measures to reduce the damage caused by typhoon conditions. It is valuable for those wind farms located in coastal areas. However, there are some deficiencies in the research that require improvement. The prediction accuracy of wind speed goes down when it comes to further future prediction. The decrease in prediction accuracy will become obvious if the actual typhoon status changes drastically, though the state of the typhoon is stable for most of the time. Therefore, future development emphasis should be placed on the enhancement of prediction accuracy for the longer term. Moreover, the method should be extended to other fields such as transmission lines to show its true value.

\section{Nomenclature}

$B: \quad$ Bias column vector

$C_{t-i}: \quad$ The correlation value of $p_{t-i}$ on the previous track of the current typhoon

$c_{\text {ita }}$ : The inertia constant

$c_{\text {ind }}$ : The constant of individual experience

$c_{\text {swm }}$ : The constant of social experience

$D: \quad$ The number of elements in matrix $W$ and $B$

$d_{m}: \quad$ The distance between $p_{m}$ and $p_{t}$ in $\mathrm{km}$

$d_{t-i}: \quad$ The distance between $p_{t}$ and $p_{t-i}$ in $\mathrm{km}$

$e_{i}$ : The relative simulation error for point $i$

$f_{c}$ : The Coriolis force parameter

E: $\quad$ Error coefficient

$e_{d}: \quad$ The MSE of wind speed direction simulation with ELM

$e_{i}: \quad$ The relative simulation error of point $i$

$e_{v}: \quad$ The mean squared error (MSE) of wind speed magnitude simulation with ELM

$F: \quad$ The fitness of an individual

$F_{\mathrm{PSO}}$ : A particle's performance

$\mathbf{F}_{t}$ : The boundary layer friction force

$F_{x}$ : The fitness value of parameter combination $x$

$G_{\text {ind }}$ : The best scheme of an individual bird based on its own searching experience 
$G_{\text {swm }}$ : The best scheme of the whole bird swarm

k: $\quad$ A unit vector

$K$ : $\quad$ The number of the output data types

$K_{C}: \quad$ The scale factor

$L_{\mathrm{ltd}, c}$ : The latitude of the wind farm center

$L_{\mathrm{ltd}, i}: \quad$ The latitude of the $i^{\text {th }}$ wind turbine in the wind farm

$L_{\operatorname{lgd}, c}: \quad$ The longitude of the wind farm center

$L_{\operatorname{lgd}, i}$ : The longitude of the $i^{\text {th }}$ wind turbine in the wind farm

$M: \quad$ The number of data types

$M_{t}$ : The total number of wind turbines in the wind farm

$M_{h}$ : The number of historical typhoon points in the influence circle

$M_{c}$ : The number of the current typhoon points in the influence circle

$N$ : $\quad$ The number of neurons in the hidden layer

$n$ : $\quad$ Population size

$\mathbf{v}_{c}$ : The typhoon wind speed

$P_{0}$ : $\quad$ The typhoon center pressure

$P_{\text {ind' }}$ : The new individual parameter scheme of a bird

$p_{\text {air }, t}: \quad$ Air pressure of the typhoon center at moment $t$ in $\mathrm{hPa}$

$P_{\text {ind }}: \quad$ The individual parameter scheme of a bird

$p_{t}$ : The position of the current typhoon at the moment $t$

$p_{t+i}$ : The position of the current typhoon in the next $i$ hour

$p_{t-i}: \quad$ The position of the current typhoon $i$ hour ago

$P_{x}$ : The probability of selection for parameter combination $\mathrm{x}$

Q: $\quad$ Output weight matrix

$R: \quad$ The radius of influence circle

$R_{\text {dec, } t}$ : The decrease limit range of the detection window at current moment $t$

$R_{\text {inc, } t}: \quad$ The increase limit range of the detection window at current moment $t$

$R_{m}$ : The maximum wind speed radius

$r_{\text {ind }}$ : The random value for individual experience

$r_{\text {swm }}$ : The random value for a social experience

$S_{m}: \quad$ Overall similarity of $p_{m}$

$S_{g, m}: \quad$ The geographic similarity of $p_{m}$ on the historical typhoon track

$S_{y, m}: \quad$ The translation speed similarity of $p_{m}$

$S_{\theta, m}: \quad$ The translation direction similarity of $p_{m}$

$v_{c}$ : Translation speed of typhoon center in $\mathrm{km} / \mathrm{h}$

$\mathbf{v}_{g}: \quad$ The gradient wind speed

$v_{m}$ : The translation speed of $p_{m}$ in $\mathrm{km} / \mathrm{h}$

$V_{\text {max }}: \quad$ The maximum wind speed among the typhoon structure in $\mathrm{m} / \mathrm{s}$

$V_{\text {max }, t}: \quad$ The maximum wind speed among the typhoon structure at moment $t$ in $\mathrm{km} / \mathrm{h}$

$v_{r, i}: \quad$ The real-time measured average wind speed at $i^{\text {th }}$ monitoring point

$v_{s, i}: \quad$ The simulated average wind speed at $i^{\text {th }}$ monitoring point

$v_{t}: \quad$ The translation speed of $p_{t}$ in $\mathrm{km} / \mathrm{h}$
$V_{\text {ind }}: \quad$ The adjustment of $P_{\text {ind }}$

$V_{\text {ind, last }}$ : The adjustment of $P_{\text {ind }}$ for the last iteration

$W: \quad$ Input weight matrix

$X: \quad$ Input data column vector

$Y$ : $\quad$ Output data column vector

$y_{t-1}$ : The wind speed magnitude of the last moment

$y_{t}$ : The wind speed magnitude of current moment $t$

$y_{k, t}: \quad$ The predicted wind speed of the current moment obtained using the Karman filter

$y_{i, f} \quad \quad$ The wind speed after filtering at moment $i$

$y_{i, u}: \quad$ The wind speed on the upper envelope line at moment $i$

$y_{i, l}: \quad$ The wind speed on the lower envelope line at moment $i$

$\Delta_{\text {inc, } t-n}$ : The increment of wind speed at moment $t-n$

$\Delta_{\mathrm{dec}, t-n}$ : The decrease of wind speed at moment $t-n$

$\Delta \lambda: \quad$ Parameter change rate set of set $\lambda_{t}$

$\Psi_{s, i}: \quad$ The simulated wind direction at $i^{\text {th }}$ monitoring point

$\rho_{a}: \quad$ The air density

$\theta: \quad$ Translation direction of typhoon center in ${ }^{\circ}$

$\theta: \quad$ The translation direction of $p_{m}$

$\theta_{t}: \quad$ The translation speed of $p_{t}$

$\theta_{r, i}: \quad$ The real-time measured wind direction at $i^{\text {th }}$ monitoring point

$\varphi_{\text {ltd }}: \quad$ Latitude of typhoon center in ${ }^{\circ}$

$\varphi_{\text {lgd }}: \quad$ Longitude of typhoon center in ${ }^{\circ}$

$\lambda_{t}: \quad$ Parameter set

$\varphi_{\text {ltd, } t}: \quad$ Latitude of typhoon center at moment $t$ in ${ }^{\circ}$

$\varphi_{\operatorname{lgd}, t}: \quad$ Longitude of typhoon center at moment $t$ in ${ }^{\circ}$.

\section{Data Availability}

The data used to support the findings of this study are available from the corresponding author upon request.

\section{Conflicts of Interest}

The authors declare that there are no conflicts of interest regarding the publication of this study.

\section{Acknowledgments}

This work was supported by the National Natural Science Foundation of China (71171081) and the Beijing Natural Science Foundation (9162014).

\section{References}

[1] S. Yuan, C. Wang, B. Mu, F. Zhou, and W. Duan, “Typhoon intensity forecasting based on LSTM using the rolling forecast method," Algorithms, vol. 14, no. 3, Article ID 83, 2021.

[2] X. Qin and W. Duan, "Forecast uncertainty of rapid intensification of typhoon dujuan (201521) induced by uncertainty in the boundary layer," Atmosphere, vol. 11, no. 11, Article ID $1263,2020$.

[3] R. Zhang, W. Zhang, Y. Zhang, J. Feng, and X. U. Liangtao, "Application of lightning data assimilation to numerical forecast of super typhoon haiyan (2013)," Journal of Meteorological Research, vol. 34, no. 05, pp. 1052-1067, 2020. 
[4] Q. Liu, J. Jiang, F. Yu et al., "Typhoon storm surge ensemble forecast based on GPU technique," Acta Oceanologica Sinica, vol. 39, no. 1, pp. 77-86, 2020.

[5] W. ChihChiang and C. C. Hsu, "Real-time rainfall forecasts based on radar reflectivity during typhoons: case study in southeastern taiwan," Sensors, vol. 21, no. 4, 2021.

[6] C.-C. Tsai and K.-S. Chung, "Sensitivities of quantitative precipitation forecasts for typhoon soudelor (2015) near landfall to polarimetric radar data assimilation," Remote Sensing, vol. 12, no. 22, Article ID 3711, 2020

[7] L.-C. Chang, F.-J. Chang, S.-N. Yang, F.-H. Tsai, T.-H. Chang, and E. E. Herricks, "Self-organizing maps of typhoon tracks allow for flood forecasts up to two days in advance," Nature Communications, vol. 11, no. 1, Article ID 1983, 2020.

[8] P.-C. Hsieh, W.-A. Tong, and Y.-C. Wang, "A hybrid approach of artificial neural network and multiple regression to forecast typhoon rainfall and groundwater-level change," Hydrological Sciences Journal, vol. 64, no. 14, pp. 1793-1802, 2019.

[9] F. Shahid, A. Zameer, and M. J. Iqbal, "Intelligent forecast engine for short-term wind speed prediction based on stacked long short-term memory," Neural Computing \& Applications, vol. 33, pp. 1-17, 2021.

[10] E. Meftah and M. Adel, "Application of long-short-termmemory recurrent neural networks to forecast wind speed," Applied Sciences, vol. 11, no. 5, Article ID 2387, 2021.

[11] X. Liu, Zi Lin, and Z. Feng, "Short-term offshore wind speed forecast by seasonal ARIMA - a comparison against GRU and LSTM," Energy, vol. 227, 2021.

[12] S. Wang, Y. Guo, Y. Wang et al., "A wind speed prediction method based on improved empirical mode decomposition and support vector machine," IOP Conference Series: Earth and Environmental Science, vol. 680, no. 1, 2021.

[13] C. Xue-Jun, J. Zhao, X. Jia, and Z. Li, "Multi-step wind speed forecast based on sample clustering and an optimized hybrid system," Renewable Energy, vol. 165, no. P1, pp. 595-611, 2021.

[14] B. Marek, C. Alexandra, and A. Dumitrescu, "Hybrid numerical models for wind speed forecasting," Journal of Atmospheric and Solar-Terrestrial Physics, vol. 220, 2021.

[15] Z. Michaël, B. Liliane, and M. Olivier, "Sequential aggregation of probabilistic forecasts-application to wind speed ensemble forecasts," Journal of the Royal Statistical Society: Series C (Applied Statistics), vol. 70, no. 1, pp. 202-225, 2020.

[16] https://www.typhoon.org.cn/.

[17] Y. Meng, M. Matsui, and K. Hibi, "An analytical model for simulation of the wind field in a typhoon boundary layer," Journal of Wind Engineering and Industrial Aerodynamics, vol. 56, no. 2-3, pp. 291-310, 1995.

[18] Y. Meng, M. Matsui, and K. Hibi, "A numerical study of the wind field in a typhoon boundary layer," Journal of Wind Engineering and Industrial Aerodynamics, vol. 67, no. 68, pp. 437-448, 1997.

[19] G. J. Holland, "An analytic model of the wind and pressure profiles in hurricanes," Monthly Weather Review, vol. 108, no. 8, pp. 1212-1218, 1980.

[20] L. An, Y. Guan, Z. Zhu, J. Wu, and R. Zhang, "Structural failure analysis of a river-crossing transmission line impacted by the super typhoon Rammasun," Engineering Failure Analysis, vol. 104, pp. 911-931, 2019.

[21] G. B. Huang, Q. Y. Zhu, and C. K. Siew, "Extreme learning machine: theory and applications," Neurocomputing, vol. 70, no. 1/3, pp. 489-501, 2006.

[22] T. Karpagalingam and M. Karuppaiah, "A hybrid approach for text document clustering using Jaya optimization algorithm," Expert Systems with Applications, vol. 178, 2021. 